\title{
Road Sign Segmentation and Recognition under Bad Illumination Condition using Modified Fuzzy C-means Clustering
}

\author{
Zinat Afrose \\ Lecturer \\ Department of Computer Science and Engineering, \\ Jahangirnagar University, Savar, Dhaka-1342, \\ Bangladesh.
}

\author{
Md. Al-Amin Bhuiyan \\ Phd, Professor \\ Department of Computer Science and Engineering, \\ Jahangirnagar University, Savar, Dhaka-1342, \\ Bangladesh.
}

\begin{abstract}
In this paper, we present a novel approach on road sign segmentation under bad lighting condition employing a modified fuzzy c-means clustering. Most of the cases accidents occur in bad weather or when the road signs cannot be recognized. The proposed system implements a system that segments the road sign using fuzzy c-means clustering. At first, the image is detected in RGB colour space and then converted into HSV colour model. After the conversion we detected the edge by applying Canny edge detector. Thus the proposed method is applied on the image. The method is based on the local similarity measure so that the noise created or the blurred image can easily be detected. But the traditional Fuzzy C-means clustering lack enough robustness to noise and preserving details of the image. Experimental results demonstrate that the system can segment the road signs successfully under bad illumination conditions. This system can be applied to typical type of traffic signs such as triangular, circle etc. The output result of the system is encouraging as the accuracy rate is $99 \%$.
\end{abstract}

\section{General Terms}

Pattern Recognition

\section{Keywords}

Clustering, Fuzzy C-means clustering, Segmentation.

\section{INTRODUCTION}

The road sign segmentation and recognition system is an important and essential aspect of "Driver Support System (DSS)", since road signs lead the drivers to drive their vehicles safely and dictates the ways, speeds and turns. But in recent years, accidents have become a serious problem in social life. Human lives are becoming uncertain day by day. Since traffic intensity is increasing day by day worldwide, roads are occupied by overloaded vehicles. But one of the main reasons of accidents is for carelessness of the drivers. Most of the cases, they are not aware of the traffic signs. In many cases, accidents occur during bad weather or in occlusion. Drivers could not identify the road signs due to bad weathers. Shadows, at night time and cloudy weather are some difficulties that drivers face. Recently many techniques have been discovered to detect road signs for safe journey. Many of them adopted to segment the road sign using the shape and colour information. For example, Piccioli et al. [1] use colour and a priori information to limit the possible locations of signs in the image. They then extract edges and look for circular or triangular regions before applying a crosscorrelation technique for recognizing the signs. In [2], a redness measure is used to locate stop, yield, and "do not enter" signs. This step is followed by edge detection and shape analysis to identify the sign. In their approach to detecting stop signs, Yuille and his colleagues [3] correct for the colour of the ambient illumination, locate the boundaries of the signs and map the sign into a front to parallel position before reading the sign. Another paper that describes actively controlling the camera for sign detection is [4]. They predict the location of the sign and point the camera for a closer view. Signs are recognized and their contents are read by template matching. A decision tree method is used in [5] to detect and recognize signs without using colour. Detection is based on shape using local orientations of image edges and hierarchical templates. The results are sent to a decision tree which either labels the regions by sign type or rejects them. A method to detect speed limit signs is given in [6]. It is based on first using colour to locate candidate signs, followed by a multiresolution application of templates that look for circular regions. Another approach is taken by Fleischer et al., [7], who use a model-based, top down approach. Extracted regions are matched using Markov model [8]. In [9], road-sign recognition method is based on image segmentation and joint transform correlation (JTC) with the integration of shape analysis. The problem was that the algorithm failed to detect road signs in the case of insufficient contrast with background and considerable occlusion. Robust road sign segmentation is done using adaptive threshold in [10]. This paper is done only for recognition of road signs for bad conditions. It has almost 98\% accuracy. But it had some fallacies also. It could not identify the cloudy or snowy road signs. In this paper, we are using Fast generalised fuzzy c-means clustering for better segmentation of the road signs. It is clear that segmentation is not easy in bad conditioned images. Most of the cases segmentation fails when the images are taken at night time, when images are obscure and when the lights are not properly controlled as shown in Figure 1. There are some major factors that cause the segmentation more difficult. Such as, signs with multiple objects surrounded, washout colour and damaged 
caused by the sunlight, not controllable light during night time. So our major concern is to detect the road sign with their colour and shape information. The fuzzy c-means algorithm is used and modified in a way that would enhance the quality of the image that is occluded with bad weather conditions. This modified Fuzzy C-means algorithm enhances the performance of the segmentation successfully. We have tested the road signs where the success rate is $99 \%$ segmentation rate.

The remainder of this paper is organized as follows. In Section 2, we present the segmentation method. The flow diagram for the proposed system is described in this section. In Section 3, the discussion about the paper and in Section 4, the experimental results and successful rates are shown. Finally, conclusions and future works are discussed in Section 5 .

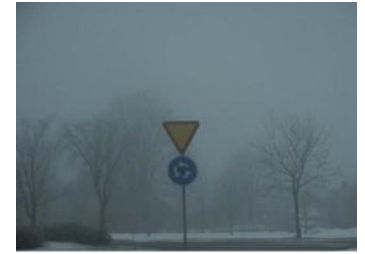

(a)

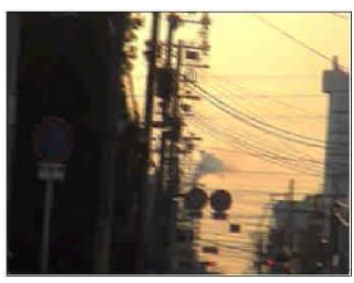

(c)

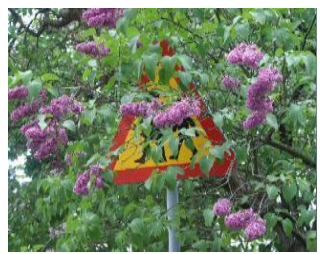

(b)

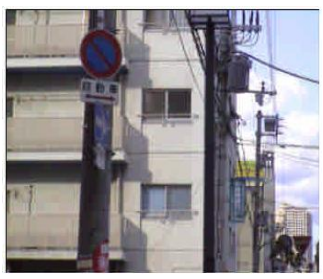

(d)
Fig 1: Images taken at different lighting conditions. (a) Snowing, (b) Obstacles, (c) Not controlled light, (d) Shadows.

\section{SYSTEM OVERVIEW}

The overall system structure is given in a flow diagram in figure 2. The road signs are detected using color information and geometric property analysis. The RGB color information is converted to HSV because The HSV colorspace is chosen because Hue is invariant to the variations in light conditions as it is multiplicative/scale invariant, additive/shift invariant, and it is invariant under saturation changes. The system is carried out by clustering the images in similar features for segmentation. The segmented regions are grouped with some attributes like aspect ratio and size. The edge is detected using Sobel operator. After the detection, the segmentation is applied using the proposed modified Fuzzy C-means clustering technique. The images are classified into different shapes like triangular, circular or rectangular shape. Novel shape classification is performed to categorize the ROIs into different shape groups and also to eliminate the false candidates that do not fall into any of the permitted shape groups. Finally, in the recognition phase, query image is compared with the corresponding to reference images in the database, and the reference sign that is most similar to the query sign is returned to the user.

\section{Region identifying and region clustering using modified fuzzy c-means clustering}

Fuzzy c-mean (FCM) is one of the most used methods for image segmentation [11] and its success chiefly attributes to the introduction of fuzziness for the belongingness of each image pixels. Compared with crisp or hard segmentation methods [12], FCM is able to retain more information from the original image. However, one disadvantage of standard FCM is not to consider any spatial information in image context, which makes it very sensitive to noise and other imaging artifacts. Fuzzy c-means (FCM) is based on minimization of the following objective function:

$J_{m}=\sum_{i=1}^{N} \sum_{j=1}^{C} u_{i j}^{m}\left\|x_{i}-c_{j}\right\|^{2}, 1 \leq m<\alpha$

where $m$ is any real number greater than $1, u_{i j}$ is the degree of membership of $x_{i}$ in the cluster $j, x_{i}$ is the $i$ th of d-dimensional measured data, $c_{j}$ is the d-dimension center of the cluster, and $\|*\|$ is any norm expressing the similarity between any measured data and the center. But for this research to detect the image in a better way a modified approach is used to detect the images in the bad illumination conditions. We introduce a special factor $S i j$ incorporating both the local spatial relationship (called $S s_{-} i j$ ) and the local gray level relationship (called $S g_{-} i j$ ) to replace the parameter $\alpha$ and make it play a more important role in clustering. Its definition is presented as below:

$$
S_{i j}=\left\{\begin{array}{cc}
S_{s_{-} i j} \times S_{g_{-} i j} & j \neq i \\
0 & j=i
\end{array}\right.
$$

where the $i$ th pixel is the center of the local window (for example, $3 \times 3$ ) and $j$ th pixel are the set of the 10 neighbors falling into a window around the $i$ th pixel. $S s_{-} i j$ makes the influence of the pixels within the local window change flexibly according to their distance from the central pixel and thus more local information can be used. Here the definition of $S s_{-} i j$ is given as in equation (3). 


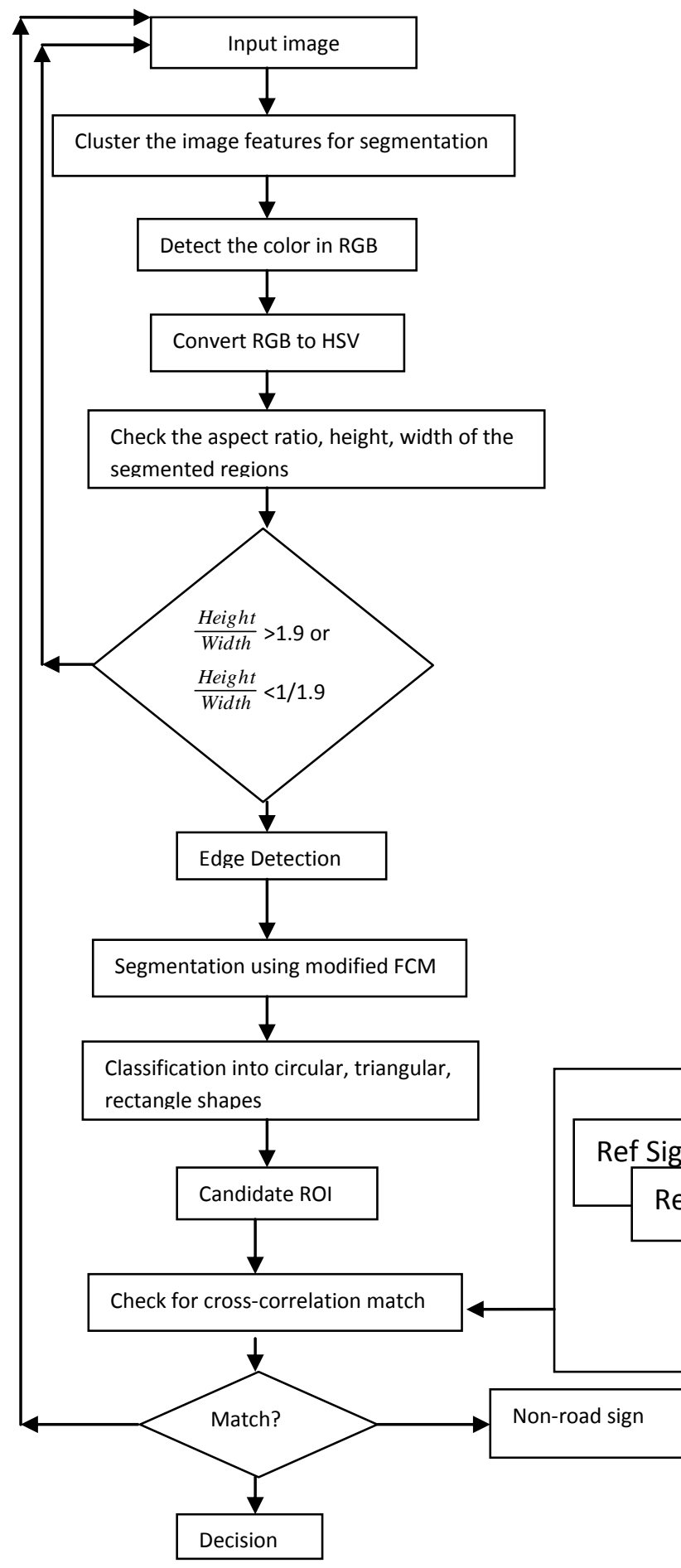

Fig 2: Flow Diagram of the proposed approach

relatively easily determined. It is worth indicating that the shape of local window defined here is square, however, the windows with other shapes such as diamond can also naturally be adopted in our algorithm. We define the local gray level similarity measure $S g \_i j$ as follows:

$$
S_{g_{-} i j}=\exp \left(\frac{-\left\|x_{i}-x_{j}\right\|^{2}}{\lambda_{g} \times \sigma_{g_{-} i}^{2}}\right)
$$

where $\sigma_{g_{-} i}$ is defined by

$$
\sigma_{g_{-} i}=\sqrt{\frac{\sum_{j \in N_{i}}\left\|x_{j}-x_{i}\right\|^{2}}{N_{R}}}
$$

where $x i$ is gray value of the central pixel within a special window, $\boldsymbol{x} j$ is gray value of the $j$ th pixels in the same window, and $\lambda g$ denotes the global scale factor of the spread of $S g_{-} i j$ and plays a similar role to $\lambda s$. The parameter $\sigma_{g_{-} i}$ is a function of the local density surrounding the central pixel and its value reflects gray value homogeneity degree of the local window. The smaller its value is, the more homogenous the local window is, and vice versa. The motivation behind the use statistics varying with each image pixel. The auto-determined parameter $\sigma_{g{ }_{-} i}$ can be computed in advance. Obviously, the $S g \_i j$ s can change automatically with different gray levels of the pixels over an image and thus reflects the damping extent in gray values.

\section{Modified Fuzzy C-Means (MFCM) Algorithm}

$S_{s_{-} i j}=\exp \left(\frac{-\max \left(\left|p_{j}-p_{i}\right|,\left|q_{j}-q_{i}\right|\right.}{\lambda_{s}}\right)$

where ( $p i, q i)$ is a spatial coordinate of the $i$ th pixel and we assume one pixel is one unit length in the above computation. $\lambda s$ denotes the scale factor of the spread of $S s_{-} i j$, determining the change characteristic of $S s_{-} i j$, thus its varying range is
Step1. 1) Set the number $c$ of the cluster prototypes change from 2 to $C_{\max }$ (predefined or set by some validity criterion or a priori knowledge);

2) Initialize randomly those prototypes and set $\varepsilon>0$ to a very small value.

Step2. Compute the local similarity measures Sij using (9) for all neighbor windows over the image. 


$$
S_{i j}=\left\{\begin{array}{cc}
S_{s_{-} i j} \times S_{g_{-} i j} j \neq i \\
0 \quad j=i
\end{array}\right.
$$

Step3. Compute linearly-weighted summed image $\xi$ in terms of (7).

$$
\xi_{i}=\frac{\sum_{j \in N i} S_{i j} x_{j}}{\sum_{j \in N i} S_{i j}}
$$

Step4. Update the partition matrix using (8).

$$
u_{i l}=\frac{\left(\xi_{l}-v_{i}\right)^{-\frac{2}{m-1}}}{\sum_{j=1}^{c}\left(\xi_{l}-v_{j}\right)^{-\frac{2}{m-1}}}
$$

Step5. Update the prototypes using (9).

$$
v_{i}=\frac{\sum_{l=1}^{q} \gamma_{l} u_{i l}^{m} \xi_{l}}{\sum_{l=1}^{q} \gamma_{l} u_{i l}^{m}}
$$

Repeat Steps4-5 until the following termination criterion is satisfied:

$\mid$ Vnew - Vold $\mid<\varepsilon$, where $V=[v 1, v 2, \ldots, v c]$ are the vectors of cluster prototypes.

\section{EXPERIMENTAL RESULT}

To evaluate the proposed approach, we have performed experiments on a large collection of images, where some test images are obtained from Google image and some real image. In fig.3, the algorithm used in this research is applied on different images where the images are taken at different lighting conditions like snowfall, rain and fog.

The road signs are then detected using their aspect ratio and sizes. After the ROI is located after applying MFCM, the detected image is compared with the road signs stored in database as shown in fig.4.

Table 1 Success rate of segmentation for the road signs

\begin{tabular}{|l|c|c|}
\hline Effect & $\begin{array}{l}\text { No. of } \\
\text { signs }\end{array}$ & MFCM algorithm \\
\hline $\begin{array}{l}\text { Bad } \\
\text { lighting }\end{array}$ & 15 & 98.5 \\
\hline $\begin{array}{l}\text { Blurred } \\
\text { image }\end{array}$ & 14 & 99 \\
\hline Dusk/dawn & 13 & 97.6 \\
\hline $\begin{array}{l}\text { Faded } \\
\text { signs }\end{array}$ & 10 & 90 \\
\hline Fog & 17 & 95.5 \\
\hline $\begin{array}{l}\text { Noisy } \\
\text { image }\end{array}$ & 25 & 98.4 \\
\hline Rain fall & 23 & 97 \\
\hline Snowfall & 15 & 98 \\
\hline Sunny & 16 & 100 \\
\hline
\end{tabular}

Segmentation accuracy \% of the three algorithms on road sign image

\begin{tabular}{|l|c|c|c|}
\hline & FCM & FKM & MFCM \\
\hline $\begin{array}{l}\text { Mixed } \\
\text { noise } \\
\alpha=0.3\end{array}$ & 95.80 & 93.30 & 97.75 \\
\hline $\begin{array}{l}\text { Mixed } \\
\text { noise } \\
\alpha=0.5\end{array}$ & 99.44 & 98.48 & 99.84 \\
\hline $\begin{array}{l}\text { Mixed } \\
\text { noise } \\
\alpha=0.7\end{array}$ & 99.69 & 99.59 & 99.96 \\
\hline
\end{tabular}



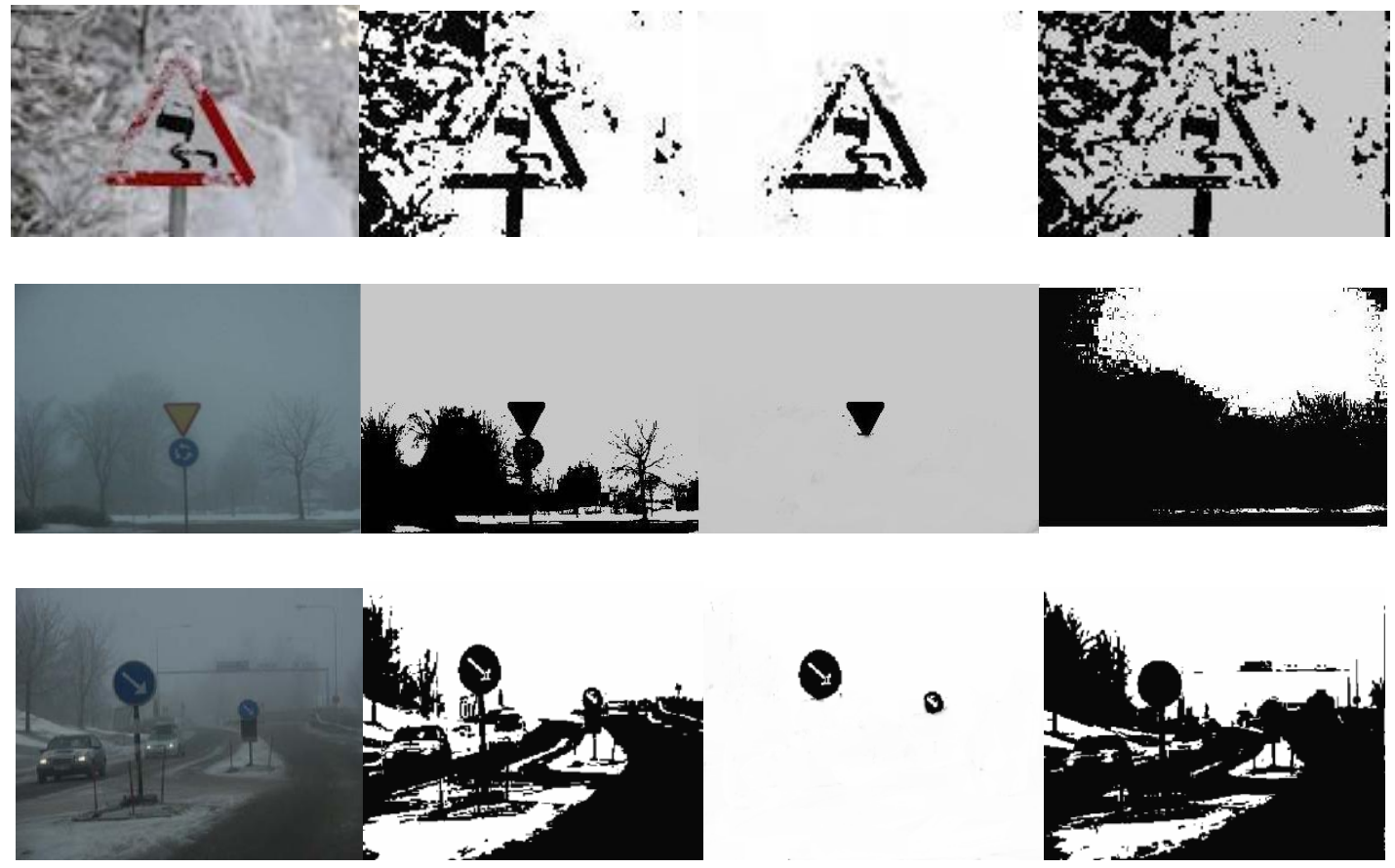

Fig 3: Segmentation result. First column: Original images. Second column: Segmented images using MFCM algorithm. Third column: Candidate ROIs. Fourth column: Segmented images using FCM algorithm.

After the occluded road sign is detected from the database, the system shows the real road sign. Hence though the lighting conditions are not good enough, the road signs can easily be detected applying this approach. The segmentation success rate $(\%)$ under different effects using Modified Fuzzy c-means clustering is shown below in Table 3. Table 4 gives the Segmentation accuracy (SA) of three algorithms on different noisy images with different levels. From table 3 we can see that the proposed method - the MFCM algorithm give rise to better denoising performance than the K-means algorithm and the classic Fuzzy C-means algorithm. It shows the success rate on the road sign images using some noise factor when $\alpha=0.3$, $\alpha=0.5, \alpha=0.7$, applying three algorithms-Fuzzy C-means clustering, Fuzzy K-means clustering and Modified Fuzzy Cmeans Clustering.

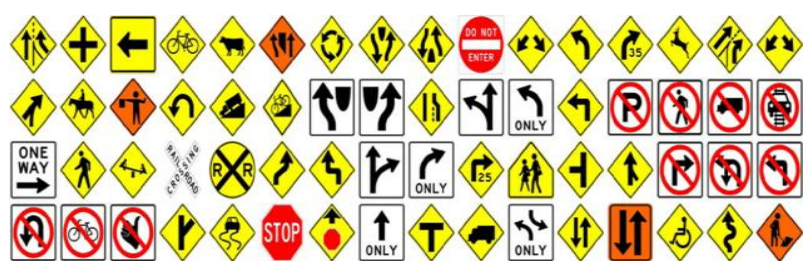

Fig.4: Some example reference images from database.

\section{CONCLUSION}

The proposed system can detect the road signs properly. The system has been tested on different road signs around the world and found good result. This research proposes a system to detect and recognize the road signs such that the road signs can easily be identified and be followed. The main contribution is that it introduces the Fuzzy c means clustering for better performance. The segmenting time is dependent only to the number of gray level q rather than size $N(>>$ ) of the image in our proposed algorithm. So the computational complexity is reduced from $\mathrm{O}\left(\mathrm{NcI}_{I}\right)$ to $\mathrm{O}\left(q c I_{2}\right)$, where $c$ is the number of clusters and $I_{1}$ and $I_{2}\left(<I_{1}\right)$ are the number of iterations respectively in the Fuzzy c-means algorithm and the proposed Modified Fuzzy c-means algorithm.

\section{REFERENCES}

[1] Piccioli, G., De Micheli, E., Parodi, P., and Campani, M., "A Robust Method for Road Sign Detection and Recognition," Image and Vision Computing, vol. 14, no. 3, pp. 209-223, 1996.

[2] L. Estevez and N. Kehtarnavaz, "A real-time histographic approach to road sign recognition," in Proc. IEEE Southwest Symp. Image Analysis Interpretation, pp. 94100, 1996.

[3] Yuille, A. L., Snow, D., and Nitzberg, M. Signfinder, "Using Color to Detect, Localize and Identify Informational Signs," in Int. Conf. on Computer Vision (ICCV98), Bombay, India, pp. 628-633, 1998.

[4] Miura, Jun, Tsuyoshi, Kanda, and Shirai, Yoshiaki, "An Active Vision System for Real-Time Traffic Sign Recognition," IEEE Conf. on Intelligent Transportation Systems, Dearborn, MI, pp. 52-57, 2000.

[5] Paclik, Pavel and Novovicova, Jana, "Road Sign Classification Without Color Information," in Proc. of the 6th Conf. of Advanced School of Imaging and Computing, Lommel, Belgium, 2000.

[6] Sekanina, Lukas and Torresen, Jim, "Detection of Norwegian Speed Limit Signs," in Proc. of the 16th European Simulation Multiconference (ESM-2002), Darmstadt, Germany, pp. 337-340, 2002.

[7] Fleischer, K, Nagel, H.-H., and Rath, T. M., "3DModelBased-Vision for Innercity Driving Scenes," IEEE Intelligent Vehicles Symp. (IV'2002), Versailles, France, 
pp. 477-482, 2002. A. de la Escalera and L. Moreno, "Road traffic sign detection and classification," IEEE Trans. Industrial Electromagnetism, vol. 44, pp. 847859, 1997.

[8] M. Lalonde and Y. Li, "Detection of road signs using color indexing," Centre de Recherche Informatique de Montreal, Montreal, QC, Canada, CRIM-IT-95, pp. 1249, 1995.

[9] J.F.Khan, S.M.A. Bhuiyan, R.R. Adhami, "Image Segmentation and Shape Analysis for Road-Sign Detection", IEEE Transactions on Intelligent Transportation Systems archive,Volume 12 Issue 1, March 2011 ,Page 83-96.
[10] Chung-Hsin Liao , Hiromitsu Hama, "Robust Road Sign Segmentation," IEICE Electronics express, Vol.2, No.14, 423-428, 2005.

[11] G. Jiang and T. Choi, "Robust detection of landmarks in color image based on fuzzy set theory," presented at Fourth Inter. Conf. on Signal Processing, Beijing, China, 1998.

[12] D. Ghica, S. Lu, and X. Yuan, "Recognition of traffic signs by artificial neural network," presented at IEEE Inter. Conf. Neural Networks, Perth, W.A., 1995. 